\title{
A Comparison Between Colour and Luminance Contrast in a Spatial Linking Task
}

\author{
FRED KINGDOM,*§ BERNARD MOULDEN, $\uparrow$ SANDRA COLLYER
}

Received 10 July 1990; in revised form 17 June 1991

\begin{abstract}
We report experiments which compare the ability of subjects to employ colour vs luminance contrast as a basis for discriminating the degree of collinearity of random element string pairs. The purpose of the study was to determine the extent to which spatial integration mechanisms could utilize colour contrast. In order to probe directly the processes of spatial integration per se, it was necessary to control for any differences in the efficiency with which the visual system utilized colour and luminance contrast to locate the positions of the individual elements in the test stimuli. To do this we first established the "equivalent" luminance contrast of an isochromatic stimulus which produced equal performance to an isoluminant stimulus in a 2 element per string alignment task. This equated the colour defined and luminance defined stimuli for local positional acuity. We then measured performance for both isoluminant and equivalent luminance contrast stimuli for strings consisting of 2, 4, 8 and 16 elements. This tested for any differences in the processes of spatial integration. For both unmasked stimuli and stimuli embedded in luminance noise, there was no consistent trend favouring either luminance or colour contrast as the number of elements in the stimuli was increased. We conclude that the visual system is able to employ colour contrast as efficiently as luminance contrast for collinearity judgements, thus implicating a general role for colour vision in spatial integration tasks.
\end{abstract}

Colour contrast Luminance contrast Isoluminance Spatial integration

\section{INTRODUCTION}

The role that colour vision plays in the processing of spatial information is currently a subject of intense interest (Mollon, 1989; Mullen \& Kingdom, 1991). In this communication we examine the role of colour vision in the process of spatial integration. We use the term spatial integration to describe any process by which structural information is obtained from multi-element stimulus patterns. The question of whether colour vision can subserve spatial integration tasks has aroused some controversy in the literature (Cavanagh, 1987; Troscianko, 1987; Livingstone \& Hubel, 1987, 1988; Mollon, 1989; Zrenner, Abramov, Akita, Cavey, Livingstone \& Valberg, 1990; Mullen \& Kingdom, 1991). For example, reports of difficulty in achieving figure-ground segregation in isoluminant line drawings (Livingstone \& Hubel, 1988) and the apparent perceptual break up of complex patterns such as the Fraser spiral at iso-

*Vision Research Center, Department of Ophthalmology, Royal Victoria Hospital Rm H4-14, 687 Pine Avenue West, Montreal, Quebec, Canada H3A 1A.

†Department of Psychology, University of Western Australia, Nedlands, Perth, WA 6009, Australia.

$¥$ Department of Psychology, University of Reading, Building 3, Earley Gate, Whiteknights, Reading RG6 2AL, England.

$\S$ To whom all correspondence should be addressed. luminance (Gregory, 1977), has led some investigators to suggest a reduced role for colour in spatial integration or "linking" tasks (Livingstone \& Hubel, 1987, 1988; Zrenner et al., 1990). On the other hand, Troscianko (1987) finds no loss in performance at isoluminance for detecting mirror symmetry in random element patterns defined solely by colour contrast.

In order to evaluate whether or not colour vision plays a reduced role in spatial integration tasks, it is first necessary to make two caveats. The first concerns the status of the claim that a minimum in performance at isoluminance in a given task implicates a reduced ability of the visual system to employ colour contrast for that task. The empirical result is usually obtained by measuring performance with a heterochromatic stimulus at a variety of luminance contrasts in and around the isoluminant point. Performance minima at isoluminance have been widely demonstrated in a variety of tasks, e.g. stereopsis in random-dot stereograms (Lu \& Fender, 1972; De Weert, 1979), vernier acuity (Morgan \& Aiba, 1985), perceived velocity (Moreland, 1980; Cavanagh, Tyler \& Favreau, 1984) and apparent motion (Ramachandran \& Gregory, 1978; Troscianko, 1987). Such performance minima however would be expected to occur even if colour contrast and luminance contrast contributed equally (though additively) to performance, since at isoluminance, luminance contrast has been 


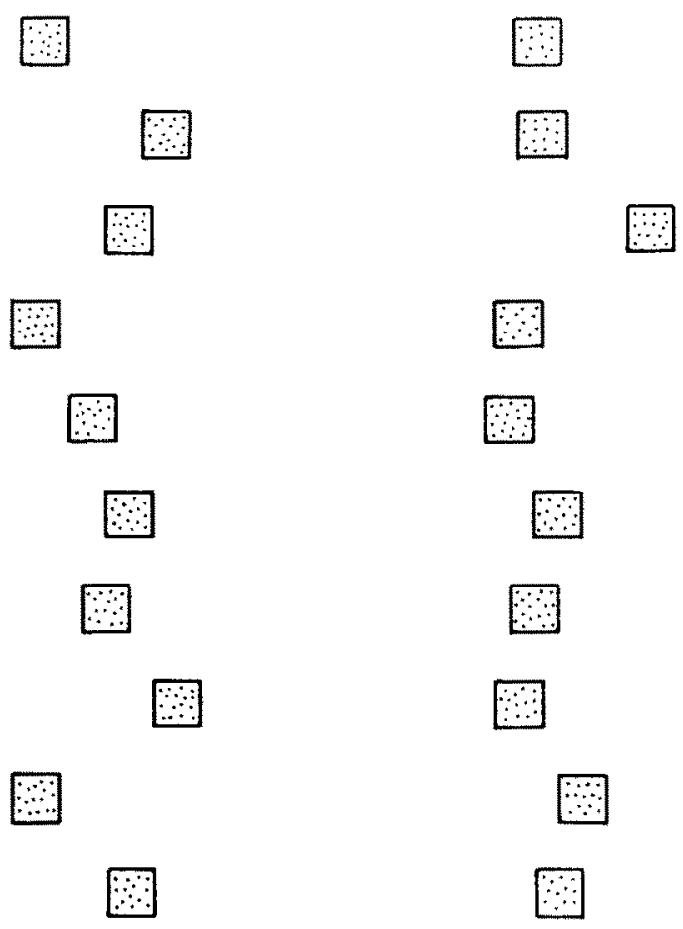

FIGURE 1. The collinearity discrimination task. Subjects had to judge on each trial which string appeared straighter or more collinear.

removed from a stimulus that contains both luminance contrast and colour contrast. To compare colour and luminance in a given task, isoluminant (colour contrast only) and isochromatic (luminance contrast only) stimuli must be equated in some way, for example by setting the contrasts of the stimuli to equal multiples of their individual detection thresholds (Troscianko \& Harris, 1988; Krauskopf \& Farrel, 1991).

Secondly, it is intuitively obvious that a perceptual representation of the spatial structure of a multi-element stimulus requires that the locations of the individual elements be first encoded. If it were the case that positional acuity was worse for isoluminant than isochromatic stimuli, then one would expect performance in spatial integration tasks to be poorer for colour defined compared with luminance defined patterns. This however could not be attributed to an impoverishment in the ability of the visual system to employ colour contrast for the process of spatial integration per se. Higher vernier alignment thresholds for colour defined compared with luminance defined stimuli have been demonstrated for spatially broad-band stimuli (Morgan \& Aiba, 1985; Krauskopf \& Farrell, 1991), though for narrow-band stimuli the results are equivocal, with Troscianko and Harris (1988) finding worse performance for colour with gratings, while Krauskopf and Farrell (1991) find no differences using Gabor patches. In the above experiments on vernier acuity the confounding effects of stimulus contrast were either shown not to be the salient factor (Morgan \& Aiba, 1985) or were controlled for (Troscianko \& Harris, 1988; Krauskopf \& Farrell, 1991). Given the possibility of poorer positional acuity with chromatically defined compared with luminance defined stimuli, the demonstration that spatial integration tasks are performed worse with chromatically defined stimuli requires that any effects of positional acuity must be controlled for.

The aim of this study is to measure psychophysical performance for a spatial integration task in order to answer the following question. Is performance worse for isoluminant compared with isochromatic stimuli when any effects of local positional acuity have been controlled for?

The spatial linking task we have chosen for this investigation is illustrated in Fig. 1. The stimulus consists of two strings of elements whose individual locations are randomly generated on each trial according to rules described below. The task for the subject on each trial was to decide which string was straighter, or more collinear. The parameters we varied included the collinearity difference between the string pairs, the colour and luminance contrast of the elements with respect to their background, and the number of elements in each string. A brief account of this study has been given elsewhere (Kingdom, Moulden \& Collyer, 1990).

\section{METHOD}

\section{Apparatus and calibration}

All stimuli were generated by an 8 bit PLUTO II graphics display system interfaced to a CORVUS CONCEPT host computer. The programs were written in Pascal using ASM68K assembly language subroutines to interface the host and graphics computers. The stimuli were displayed on a MICROVITEC CUB model 1449 RGB video monitor. All luminance calibrations were made with a United Detector Technology microphotometer, Model 81, focused onto a small patch of pixels whose RGB value had been preset. Each pixel measured $0.61 \mathrm{~mm}$ in height and $0.38 \mathrm{~mm}$ in width, subtending 0.51 and 0.32 arc min respectively at the viewing distance of $414 \mathrm{~cm}$ employed throughout the experiments. The video screen measured $4.03 \mathrm{deg}$ in width by $2.44 \mathrm{deg}$ in height. The colour coordinates of the $\mathrm{R}$ and $\mathrm{G}$ channel phosphors on the video monitor were $\mathrm{R} x=0.625$, $y=0.34 ; \mathrm{G} x=0.31, y=0.592$. In all the experiments the stimulus elements employed were either "green" $(x=0.34, y=0.31)$ or "yellow" (approx. $x=0.51$, $y=0.43)$.

\section{Subjects}

For the main part of the study three subjects SC, KC and FK took part, and a fourth, CB, participated in the first experiment. SC and FK were experienced psychophysical observers. CB and $\mathrm{KC}$ were naive as to the purpose of the experiments. All had uncorrected normal vision and passed $100 \%$ on Stilling's pseudoisochromatic plates. In the final experiment a group of 9 undergraduate students were employed. All were naive as to the purpose of the experiment. All had normal or uncorrected vision. Because of the nature of the task, the practice trials in the experiment served to screen the 
subjects for any colour vision deficits, and all subjects performed above $95 \%$, indicating that they had normal colour vision.

\section{Measure of collinearity difference and method of stimulus generation}

To measure the collinearity of an $N$ element string along its vertical axis we define the parameter $J$ as the average horizontal separation between all possible pairs of elements within the string. This requires a total of $N(N-1) / 2$ comparisons and thus

$$
J=\frac{2}{N(N-1)} \sum_{i=1}^{N} \sum_{j=i}\left|x_{j}-x_{j}\right|
$$

here $x_{i}$ and $x_{j}$ are the horizontal coordinates of the $i$ th and $j$ th elements in the string respectively. We define the collinearity difference for each string pair as $\Delta J$, the difference in their average horizontal between element separation. For each stimulus an iterative search was carried out on randomly generated sets of $N$ horizontal coordinate values until a pair of sets was found whose computed value of $\Delta J$ fell within a specified window. The value of $\Delta J$ for that condition was defined as the mid-point in the window. The only constraint that was imposed on this procedure was the maximum allowable value of $\left|x_{i}-x_{j}\right|$, that is the maximum allowable width of the stimulus as a whole.

\section{Measure of luminance contrast}

For the experiments using unmasked stimuli, the luminance contrast $C$ of the stimulus was defined as

$$
C=\log \left(L_{\mathrm{t}} / L_{\mathrm{b}}\right)
$$

where $L_{\mathrm{t}}$ and $L_{\mathrm{b}}$ were the luminances in candelas per square metre of the test stimulus and background respectively. For the experiments employing masking luminance noise, contrast was defined as the difference in mean luminance $\Delta L$ between the stimulus and the background.

\section{Procedure for main experiments}

For all experiments subjects viewed the stimuli monocularly through a $2 \mathrm{~mm}$ artificial pupil which served to reduce chromatic aberration. For the first two experiments, subjects were required at the start of the experimental session to adjust their head position against a back rest until the residual chromatic aberration in an isoluminant green-yellow checkerboard displayed on the screen was minimized. At any time during the experiment the checkerboard could be reintroduced onto the screen by a button press. In the last two experiments the stimuli were embedded in luminance noise to mask chromatic aberration effects and this procedure was therefore not necessary.

After sufficient practice sessions, subjects adapted to the stimulus background for $2 \mathrm{~min}$ prior to the presentation of the first stimulus. On each trial the stimuli were presented for $0.5 \mathrm{sec}$ and the subject indicated by a button press which of the two strings appeared most collinear. An audible signal was sounded when a correct response was made. The trials were self-paced, with an interval of $1 \mathrm{sec}$ between the button press and the presentation of the next stimulus.

For each stimulus condition 180 trials were presented, and performance was measured as the percent correct collinearity discriminations.

\section{Measurement of isoluminance}

We employed the method of heterochromatic ficker photometry (HFP) to establish the isoluminant points for the various stimulus conditions. A $5 \times 5$ checkerboard of green and yellow squares was set against a yellow background. The squares were the same size as the string elements employed in the main task condition, and the luminance of the yellow squares and background was fixed at $36.0 \mathrm{~cd} \mathrm{~m}^{-2}$, as in the main task condition. The checkerboard was phase reversed at approx. $20 \mathrm{~Hz}$, and the subject adjusted the luminance of the green phase until perceived flicker was minimal. The isoluminant value was taken as the mean of five settings.

\section{EXPERIMENT 1}

\section{Effect of Luminance Conirast on Collinearity Discrimination for Yellow-Green Element Strings}

In this experiment we measured collinearity discrimination as a function of the luminance of the green clements which were set against a yellow background of fixed luminance. We wished to establish whether there was a minimum in performance at the point defined as isoluminant by the method of HFP. This would confirm that HFP was a valid method of establishing the isoluminant point for collinearity discrimination.

\section{Spatial dimensions of stimuli}

The strings each consisted of 10 elements. Each element was square subtending $6.3 \times 5.1$ arc $\mathrm{min}$ and vertically separated from its nearest neighbour by 5.1 arc min, resulting in a total string length of 97 arc min. Stimulus width was restricted to 12.6 arc min. For the first part of the experiment $\Delta J$ was fixed at $1.25 \pm 0.25 \mathrm{arc} \mathrm{min}$, and for the second part of the experiment 6 levels of $\Delta J$ were employed. Pilot studies revealed that different ranges of $\Delta J$ were required for each subject to obtain a good spread of performance within the $50-100 \%$ range, and the ranges of values of $\Delta J$ employed for each subject are indicated on the abscissae of Fig. 3.

\section{Results}

Figure 2 presents the results of the first part of the experiment. For each subject percent correct performance is plotted against luminance contrast. The solid circles represent the luminance contrast determined to be isoluminant by HFP. As can be seen, three of the four subjects show a performance minimum at isoluminance. These same three subjects also show a curious and unexpected inverse $U$ shaped function as the luminance of the green elements is increased from zero (contrast $=-\infty$ ) to the isoluminant point. 

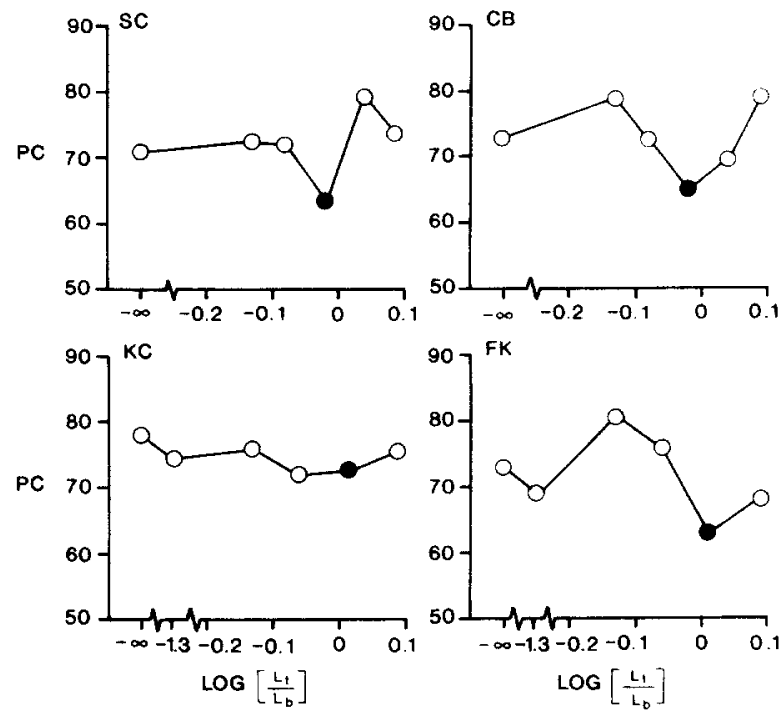

FIGURE 2. Effect of luminance contrast on collinearity discrimination. Solid circles are isoluminant contrasts as measured by HFP.

We wished to check the consistency of these measures across different values of $\Delta J$. We therefore compared two luminance contrast levels of the string pairs, $-\infty$ and isoluminance, at 6 levels of collinearity difference. The results are shown in Fig. 3. The three subjects who showed a minimum at isoluminance in the first part of the experiment show a consistent difference between the two contrast levels in this second part. One subject, $\mathrm{KC}$, who showed no performance minimum in the first part, shows no difference between the two conditions in the second part.

\section{Discussion}

These results for the most part confirm the expectation of a reduced level of performance in collinearity discrimination when luminance contrast is removed from the heterochromatic stimulus. The effects however appear not to be very large. In the second part of the experiment, the comparison between the "pure" luminance contrast condition (when the elements were at near zero luminance) and the isoluminant condition revealed an average superiority of the luminance contrast condition of about $12 \%$ across the range of $\Delta J$ employed.

Having established, at least in three of the four subjects, the presence of a performance minimum at isoluminance corresponding to the point of minimum perceived flicker as measured by HFP, it was then possible to proceed to answer directly the question we posed at the outset. Is collinearity judgement worse for isoluminant than isochromatic stimuli when the effects of local positional uncertainty have been controlled for?

\section{EXPERIMENT 2}

A Comparison Between Isoluminance and "Equivalent Luminance Contrast" Conditions on the Effect of the Number of Elements on Collinearity Discrimination

This experiment falls into two parts. In the first part, the "titration" experiment, we measured collinearity discrimination for just 2 element strings (strictly speaking the task was onc of deciding which pair of elements appeared most vertically aligned), and established for each subject a luminance contrast which produced tho same level of performance as the isoluminant condition. We refer to this luminance contrast as the "equivalent luminance contrast". Having established for each subject an equivalent luminance contrast for the $N=2$ task, we then proceeded to measure performance for 2.4 .8 and 16 element strings at both isoluminance and at the equivalent luminance contrast.

The rationale for this procedure is as follows. Performance in the 2 element string task should be influenced by factors which affect the positional acuity of the individual elements. However, the task involves the minimum of spatial integration. The positional acuity of each element should be largely unaffected by $N$, but as $N$ increases more and more spatial integration is required. If colour contrast is indeed a poor basis for linking then as $N$ is increased performance in the isoluminant condition should become increasingly worse compared with the equivalent luminance contrast condition.

\section{Part A (titration): method}

Element size and vertical inter-element separation were the same as in Experiment 1. The maximum permitted horizontal separation was 6.31 arc min and $\Delta J$ was set to $2.0 \pm 0.25$. $N$ was set to 2 elements.

Five stimulus contrasts were employed: -0.02 $-0.07,0.19,0.60$ and the isoluminant contrast established separately for each subject. The string elements were yellow in the luminance contrast stimuli and green in the isoluminant stimuli.

\section{Part A (titration): results}

Figure 4 illustrates how the equivalent luminance contrasts were established for each subject. The figures
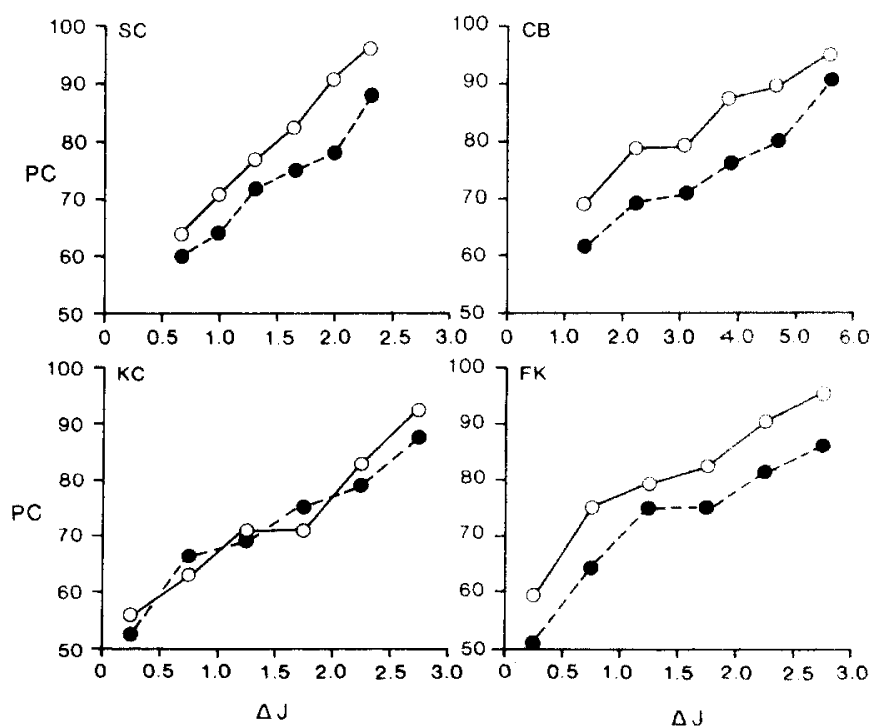

FIGURE 3. Collinearity discrimination between isoluminant (solid circles) and maximum luminance contrast (open circles) as a function of $\Delta J$, the collinearity difference. 

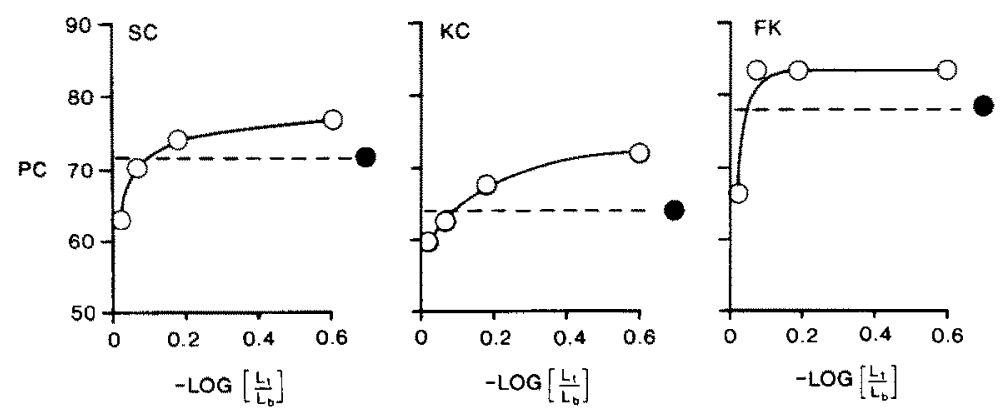

FIGURE 4. Results from the titration part of Experiment 2. Open circles represent collinearity discrimination for 2 element, monochromatic yellow, strings as a function of luminance contrast. A curve through the points is drawn by hand. Solid circles are for green yellow isoluminant conditions. The "equivalent luminance contrast" is estimated as the point on the abscissa at which the horizontal line drawn through the solid circle intersects the curve.

plot percentage correct discriminations against luminance contrast, with the continuous curves being fitted by hand through the four data points. The equivalent luminance contrast for each subject is the value on the abscissa where the horizontal line running from the isoluminant performance level intersects the curve. The equivalent luminance contrasts were estimated in this way to be $\mathrm{SC}=0.1, \mathrm{FK}=0.05$, $\mathrm{KC}=0.1$.

\section{Part B (main experiment): method}

Four string lengths were employed: $N=2,4,8$ and 16 elements. For each string length performance was measured for the isoluminant condition and the equivalent luminance contrast condition. Within each experimental session $N$ and the type of contrast (isoluminant or equivalent luminance) was randomly interleaved. The experiment was carried out for two levels of $\Delta J, \Delta J=2.0 \pm 0.25$ and $\Delta J=3.0 \pm 0.25$.

\section{Part $B$ (main experiment): results}

The results are shown in Fig. 5, which plots percent correct collinearity discriminations as a function of $N$, the number of elements in each string. Solid circles show performance for the isoluminant contrast, open circles equivalent luminance contrast, conditions. As expected, performance is now very similar for the $N=2$ conditions. The graphs do not appear to show a consistent trend in favour of luminance contrast as $N$ increases from the $N=2$ baseline.

To analyze the data we converted the percent correct scores into $d^{\prime}$ values for a 2 AFC task using Hacker and Ratcliff's (1979) revised version of Elliot's (1964) tables. The data were subjected to a within-subjects ANOVA (analysis-of-variance), with the error term in each case being the Subjects $\times$ Condition interactions (Winer, 1962). Significant main effects were found for $N[F(3,6)=5.63, P<0.05]$, and $\Delta J[F(1,2)=223$, $P<0.005]$. However the critical tests for (a) the main effect of type of contrast and (b) the interaction between type of contrast and $N$, both produced non-significant results (a) $F(1,2)=0.15, P=0.73$; (b) $F(3,6)=0.69$, $P=0.59$.

For each value of $N$ we calculated the log ratio of $d^{\prime} \mathrm{s}$ for the luminance and colour contrast conditions. These values are shown in the top panel of Fig. 9, averaged

a
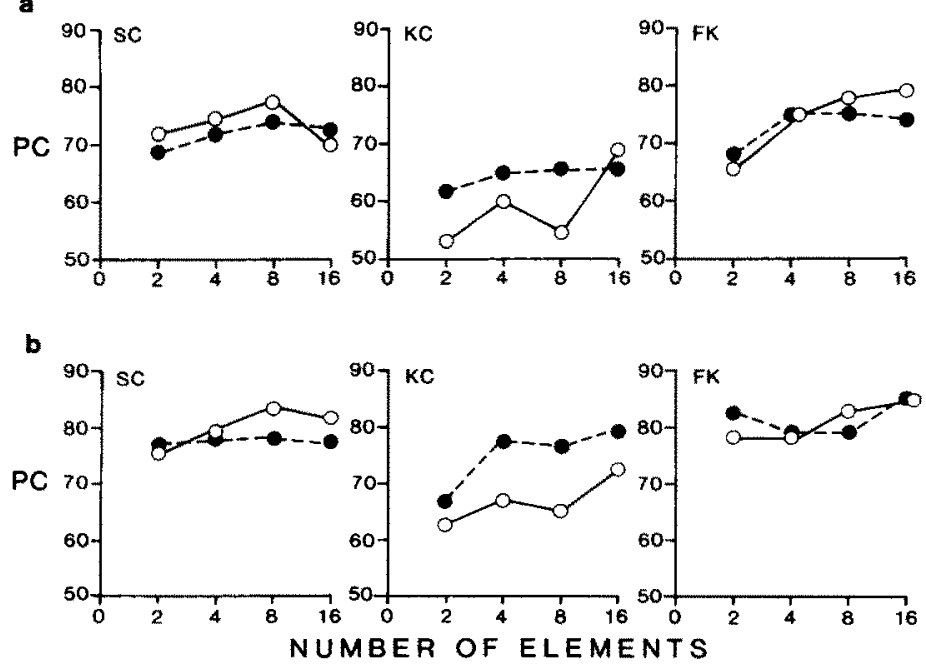

FIGURE 5. Results from main part of Experiment 2. Collinearity discrimination as a function of the number of elements, $N$, in each string. Solid circles = isoluminant condition; open circles = equivalent luminance contrast condition. (a) Lower signal strength; (b) higher signal strength condition. 
across the three subjects. As the figure shows there is a small upward trend favouring luminance contrast, and using the less conservative Page's $L$ non-parametric trend test trend is just significant though only for the larger $\Delta J$ condition $(L=84, P<0.5)$.

\section{Discussion}

The results of this experiment do not support the suggestion that colour contrast provides a poorer basis for spatial linking than luminance contrast. There is a hint of a small superiority of luminance contrast as $N$ increases in the larger $\Delta J$ condition, as revealed by the Page's $L$ test. The effect however is clearly very small, and is nowhere near significant using an ANOVA. In fact, in the larger $\Delta J$ condition, a comparison of performance between colour and luminance contrast for the $N=16$ condition (which arguably involves the greatest amount of spatial integration) shows mean percent correct values across subjects of $80.8 \%$ for the isoluminant and $79.8 \%$ for the equivalent luminance contrast conditions, a difference of $1 \%$ in favour of the colour contrast condition. Averaged across both $\Delta J$ conditions the overall difference for the $N=16$ is less than $1 \%$ in favour of the luminance contrast condition.

Although the use of an artificial pupil may reduce the effects of chromatic aberration, it does not eliminate them altogether. The presence in the retinal image of luminance "contours" on the edges of the ostensibly isoluminant stimuli may conceivably have provided a sufficiently strong input to the pathways involved in spatial integration. This would have the effect of reducing any performance differences between the colour and equivalent luminance contrast conditions compared with if there were no luminance artifacts present in the stimuli.

One way of eliminating the effects of luminance artifacts is to mask them in luminance noise. We therefore decided to repeat the above experiment with the inclusion of this feature.

\section{EXPERIMENT 3}

\section{Collinearity Discrimination in Stimuli Embedded in Luminance Noise}

An example of the stimulus employed is shown in Fig. 6. This shows an example of the equivalent luminance contrast condition for $N=16$ elements. The noise consisted of pixels $3.2 \times 2.5$ arc $\mathrm{min}$ in size (i.e. onequarter the area of a stimulus element) drawn randomly from an equal probability distribution of 8 grey levels. The 8 grey levels were on a linear grey scale spanning $18.0-40.0 \mathrm{~cd} \mathrm{~m}^{-2}$.

For the luminance contrast conditions, the target elements consisted of pixels drawn from an 8 grey level distribution spanning a range of $4.4 \mathrm{~cd} \mathrm{~m}^{-2}$. During the titration part of the experiment four contrasts were employed, contrast here being defined as $\Delta L$, the difference in mean luminance between the target and background: these values were $9,11,13$, and $15 \mathrm{~cd} \mathrm{~m}^{-2}$.
The value of $\Delta J$ employed throughout wat, 3.2 arc $\mathrm{mm}$ with a maximum permitted horizontal separation of 18.9 arc $\min$.

The Inain part of the experiment was repeated under two conditions. In the first condition different values of $N$ were randomly presented within an experimental session: this is the "unblocked" condition. In the second, the stimuli were "blocked", that is only one value of $N$ was presented within an experimental session

\section{Results}

Figures 7 and 8 show the results of this experiment. Figure 7 shows the titration results. The estimated equivalent contrasts were $\mathrm{SC}=13.5 \mathrm{~cd} \mathrm{~m}^{-2}, \mathrm{KC}=$ $12.5 \mathrm{~cd} \mathrm{~m}^{-2}, \mathrm{FK}=12.0 \mathrm{~cd} \mathrm{~m}^{-2}$.

Figure 8 shows the results of the main part of the experiment for (a) the unblocked and (b) the blocked conditions. As can be seen there appears to be no consistent trend in favour of the luminance contrast conditions as $N$ is increased. An ANOVA conducted on the data revealed significant main effects of $N$ $[F(3,6)=32, P<0.05]$ and type of stimulus presentation (blocked vs unblocked trials) $[F(1,2)=58, P<0.05]$. However, as in the previous experiment, there was no significant main effect of type of contrast $[F(1,3)=0.09$, $P=0.78]$ or significant interaction between type of contrast and $N[F(3,6)=3.2, P=0.11]$.

The bottom panel of Fig. 9 shows the results analyzed as described for the previous experiment. There is a small upwards trend in favour of luminance contrast for the unblocked trials condition, and a small downwards trend in favour of colour contrast for the blocked trials condition. A Page's $L$ test shows that the trend in the first case (unblocked trials) is just significant ( $L=86$, $P<0.05$ ). Figure 9 shows these trends.

\section{Discussion}

As in the previous experiment, there appears to be no consistent trend in favour luminance contrast as $N$ is increased. There is a hint in the data of a differential effect depending on whether the stimuli are presented in blocked or unblocked trials. This may have been the factor responsible for the small trend in favour of luminance contrast found in the previous experiment, which used exclusively unblocked trials. With unblocked trials the subjects do not know the value of $N$ on each trial and it may be that their attention is more evenly divided across the screen compared with when the exact value of $N$ is known before each presentation. This may have a differential effect on the detection of luminance as opposed to colour contrast as $N$ is increased.

\section{EXPERIMENT 4}

Population Study

We tested the generality of the broad findings obtained with the three subjects in the previous experiment by conducting a limited study on a group of naive 


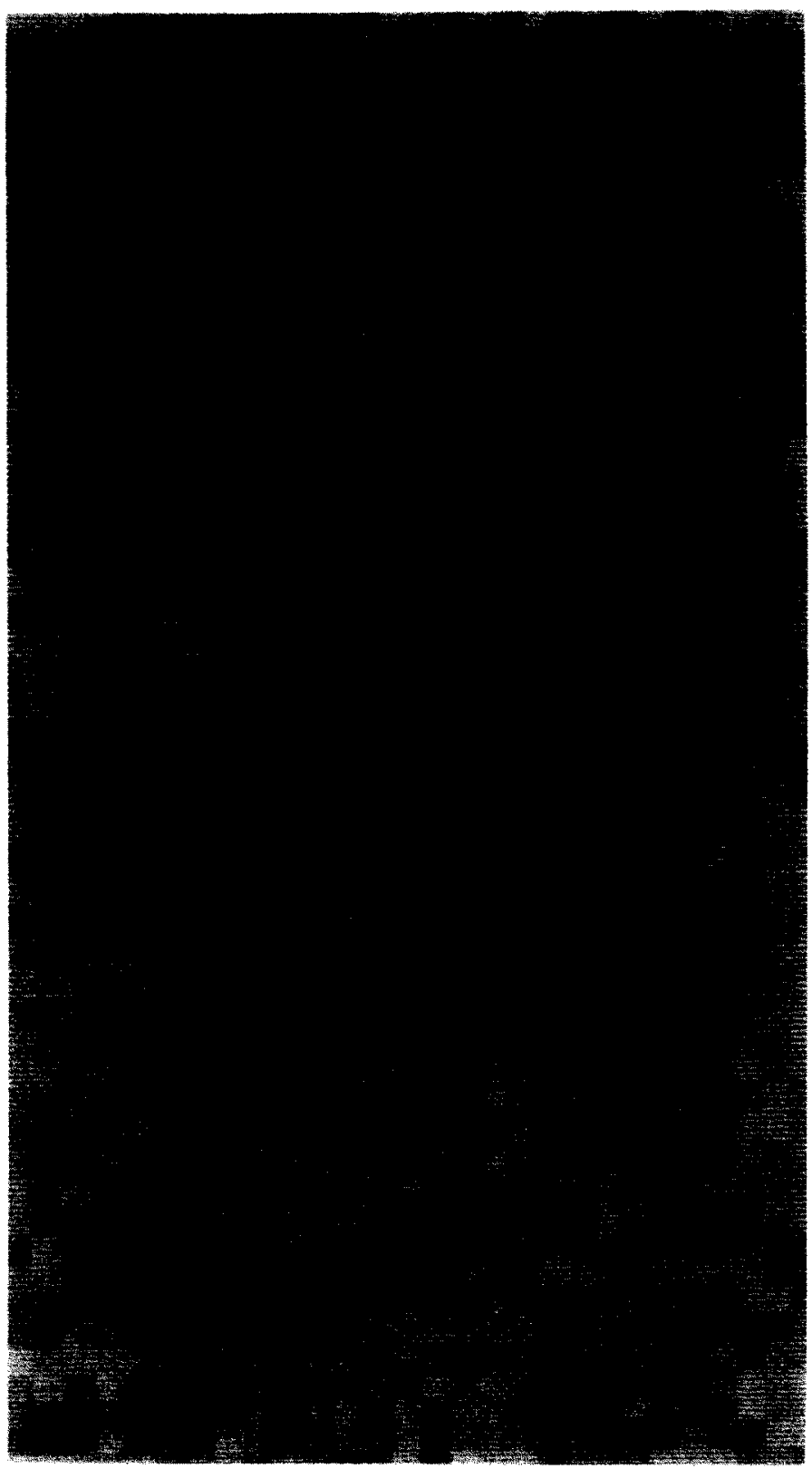

FIGURE 6. An example of an $N=16$ elements, equivalent luminance contrast stimulus embedded in luminance noise.

subjects. For each subject we measured performance for the $N=2$ and 16 conditions. We did not find a measure of equivalent contrast for each subject but used the average value obtained for the three subjects employed in Experiment 4, $12.7 \mathrm{~cd} \mathrm{~m}^{-2}$, since those subjects' indi- vidual values were very similar. Stimulus presentation employed blocked trials.

The results are shown in Fig. 10. Figure 10(a) shows the results for luminance contrast, Fig. 10(b) shows the results for colour contrast and Fig. 10(c) shows the trend
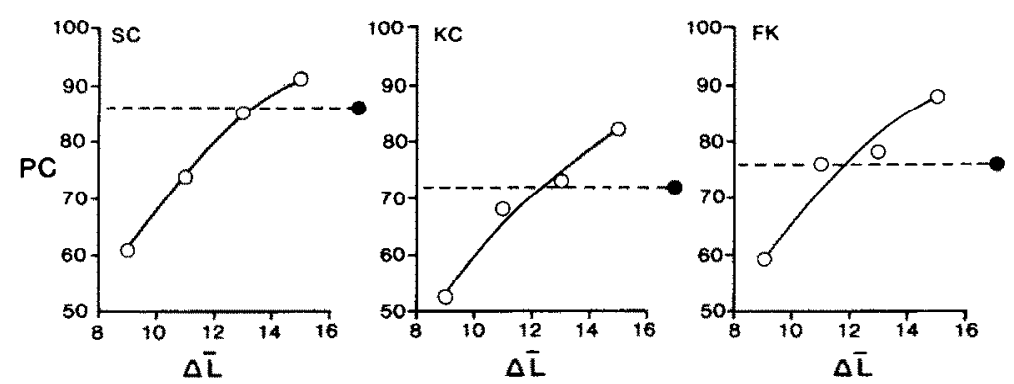

FIGURE 7. Results from titration part of Experiment 3. Symbols as in Fig. 4. 


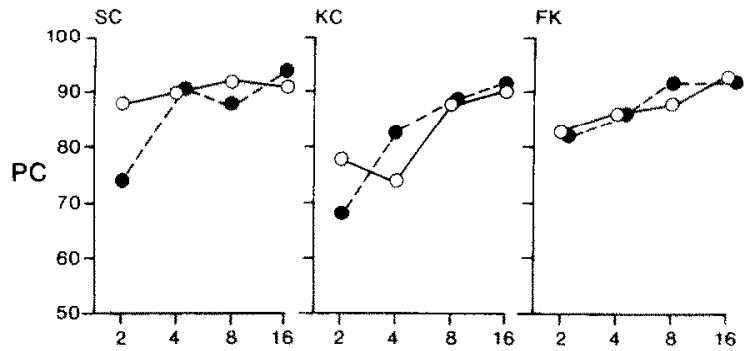

b

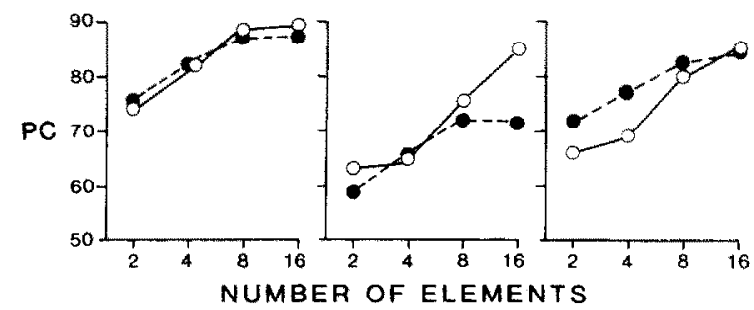

FIGURE 8. Results from main part of Experiment 4. Symbols as in Fig. 5. (a) Unblocked trials; (b) blocked trials.

in the $(\log )$ ratio between the two. As Fig. 10 shows, performance for the nine subjects appears to increase to a slightly greater extent for the colour contrast than for the luminance contrast condition as $N$ is increased from 2 to 16 elements. This difference is significant using an ANOVA to test for the interaction between $N$ and Type of Contrast $[F(1,8)=72, P<0.05]$.

\section{GENERAL DISCUSSION}

Firstly, the results of these experiments show unequivocally that colour contrast can provide an input to the pathways involved in integrating information that is inherently spatially distributed. More importantly, how-
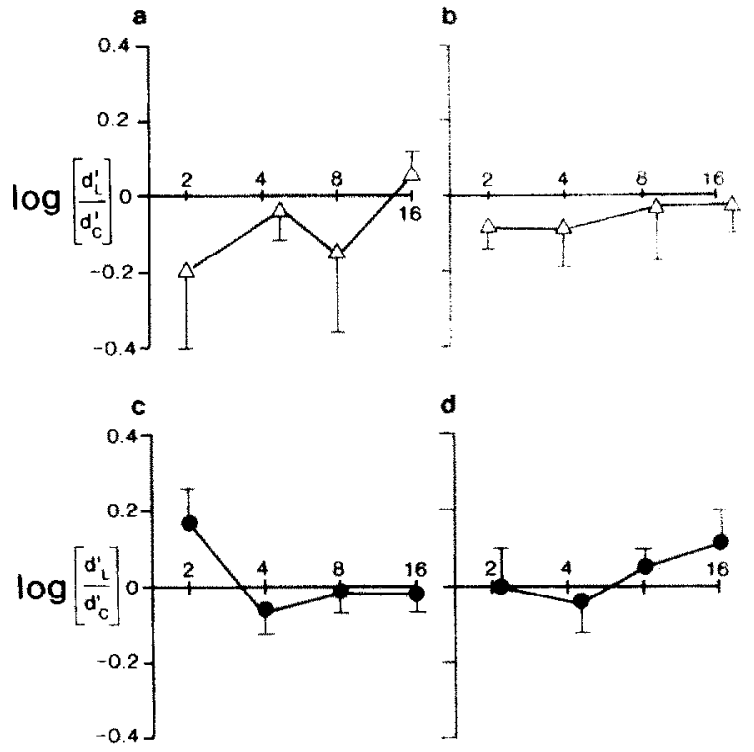

NUMBER OF ELEMENTS

FIGURE 9. Results from both Experiments 2 and 3. Mean log ratios of $d^{\prime}$ for luminance contrast and $d^{\prime}$ for colour contrast are plotted as a function of the number of elements $N$. Error bars represent standard errors across subjects. (a) Experiment 2, lower signal strength condition; (b) Experiment 2, higher signal strength condition; (c) Experiment 3, unblocked trials; (d) Experiment 3, blocked trials.

ever, they show that provided luminance contrast and colour contrast are suitably equated in their ability to encode spatial position, colour contrast is at least as good as luminance contrast for collinearity judgements. There is a hint in the data that the method of stimulus presentation (blocked vs unblocked trials) may have a differential effect favouring either luminance contrast (unblocked trials) or colour contrast (blocked trials), but the small size of the effects suggest that this factor is probably trivial.

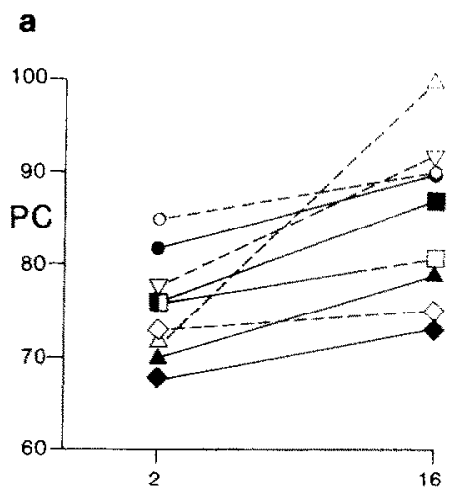

b

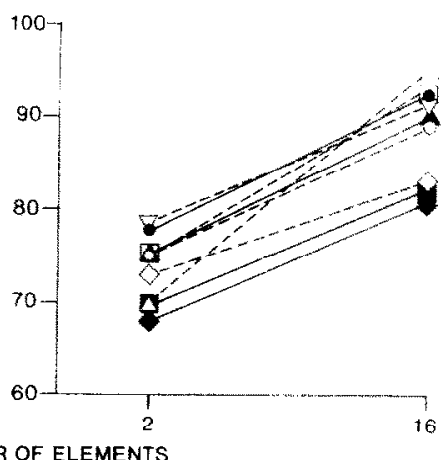

NUMBER OF ELEMENTS

c

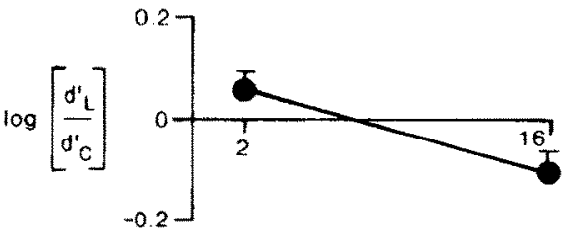

NUMBER OF ELEMENTS

FIGURE 10. Collinearity discrimination for nine naive subjects for blocked trials of stimuli embedded in luminance noise.

(a) Luminance contrast condition; (b) colour contrast condition; (c) mean (log) $d^{\prime}$ ratios. 
Given that colour contrast can provide as efficient an input as luminance contrast to spatial integration mechanisms, it is worth considering under what circumstances this might be useful for vision. In block world environments where isoluminant objects are extremely rare, the structure of virtually all objects can be obtained from the grey level representation, and the role of colour vision may be limited to that of identifying objects from their surface hue. However, in scenes such as dense foliage, colour vision may play a special role in the detection of objects and the identification of their spatial structure (Morgan, Mollon \& Adam, 1989; Mollon, 1989; Mullen $\&$ Kingdom, 1990). Because of the proliferation of shading and shadows, differences in average reflectance between overlapping clumps of foliage may become "swamped" in luminance noise and thus be undetectable. Chromatic differences would then become very useful as a potential means of perceptual segregation.

\section{REFERENCES}

Cavanagh, P. (1987). Reconstructing the third dimension: Interactions between color, texture, motion, binocular disparity and shape. Computer Graphics and Image Processing, 37, 171-195.

Cavanagh, P., Tyler, C. W. \& Favreau, O. E. (1984). Perceived velocity of moving chromatic gratings. Journal of the Optical Society of America A, 1, 893-899.

De Weert, C. M. M. (1979). Colour contours and stereopsis. Vision Research, 19, 555-564.

Drever, J. (Ed.) (1935). Stilling's pseudo-isochromatic plates for testing colour perception. Leipzig: Thieme.

Elliot, P. B. (1964). Tables of $d^{\prime}$. In Swets, J. A. (Ed.) Signal detection and recognition by human observers. New York: Wiley.

Gregory, R. L. (1977). Vision with isoluminant colour contrast: 1. A projection technique and observations. Perception, 6, 113-119.

Hacker, M. J. \& Ratcliffe, R. (1979). A revised table of $d$ ' for M-alternative forced choice. Perception and Psychophysics, 26, 168-170.

Kingdom, F., Moulden, B. \& Collyer, S. (1990). No difference between colour and luminance in the perception of collinearity. Investigative Ophthalmology and Visual Science, (Suppl.), 31, 89.
Krauskopf, J. \& Farell (1991). Vernier acuity: Effects of chromatic content, blur and contrast. Vision Research, 31, 735-749.

Livingstone, M. S. \& Hubel, D. H. (1987). Psychophysical evidence for separate channels for the perception of form, colour, movement, and depth. Journal of Neuroscience, 7, 3416-3468.

Livingstone, M. S. \& Hubel, D. H. (1988). Segregation of form, colour, movement, and depth: Anatomy, physiology and perception. Science, 240, 740-749.

Lu, C. \& Fender, D. H. (1972). The interaction of colour and luminance in stereoscopic vision. Investigative Ophthalmology, 2, 482-489.

Mollon, J. (1989). "Tho" she kneel'd in that place where they grew ..." The uses and origins of primate colour vision. Journal of Experimental Biology, 146, 21-38.

Moreland, J. D. (1980). Spectral sensitivity measured by motion photometry. In Verriest, G., (Ed.), Colour deficiencies V (Vol. 5, pp. 299-305).

Morgan, M. J. \& Aiba, T. S. (1985). Positional acuity with chromatic stimuli. Vision Research, 25, 689-695.

Morgan, M. J., Mollon, J. D. \& Adam, A. (1989). Dichromats break colour-camouftage of textural boundaries. Investigative Ophthalmology and Visual Science, 30, 220.

Mullen, K. T. \& Kingdom, F. A. A. (1991). Colour contrast in form perception. In Gouras, P. (Ed.) The perception of colour, Vol. 6 , Vision and visual dysfunction. Oxford: Macmillan.

Ramachandran, V. S. \& Gregory, R. L. (1978). Does colour provide an input to human motion perception? Nature, 275, 55-56.

Troscianko, T. (1987). Perception of random dot symmetry and apparent motion at and near isoluminance. Vision Research, 27, $547-554$.

Troscianko, T. \& Harris, J. (1988). Phase discrimination in chromatic compound gratings. Vision Research, 28, 1041-1049.

Winer, B. J. (1962). Statistical principles in experimental design. New York: McGraw-Hill.

Zrenner, E., Abramov, I., Akita, M., Cowey, A., Livingstone, M. \& Valberg, A. (1990). Colour perception: Retina to cortex. In Spillman, L. \& Werner, J. S. (Eds), Visual perception: The neurophysiological foundation. London: Academic Press.

Acknowledgement - This research was funded by an SERC (UK) Image Interpretation grant, No. GR/D 89165. 